\title{
Éditorial. Donner à lire les humanités numériques francophones (1)
}

Editorial. Francophone Digital Humanities to Read (1)

\section{(2) OpenEdition}

Journals

Édition électronique

URL : https://journals.openedition.org/revuehn/508

DOI : 10.4000/revuehn.508

ISSN : 2736-2337

Éditeur

Humanistica

Référence électronique

"Éditorial. Donner à lire les humanités numériques francophones (1) 》, Humanités numériques [En

ligne], 1 | 2020, mis en ligne le 01 janvier 2020, consulté le 15 juillet 2021. URL : http://

journals.openedition.org/revuehn/508; DOI : https://doi.org/10.4000/revuehn.508

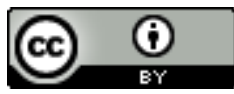

Les contenus de la revue Humanités numériques sont mis à disposition selon les termes de la Licence Creative Commons Attribution 4.0 International. 


\title{
humanités \\ numériques
}

\section{Éditorial. Donner à lire les humanités numériques francophones (1) \\ Editorial. Francophone Digital Humanities to Read (1)}

\author{
Entrées d'index \\ MOTS-CLÉS : humanités numériques, histoire des humanités numériques \\ KEYWORDS: digital humanities, history of the digital humanities
}

La création d'Humanités numériques s'inscrit dans un moment particulier des sciences humaines et sociales. Les révolutions proclamées et les transitions programmées font place à des évolutions collectives, qu'il nous appartient d'influencer pour qu'elles soient intelligentes et heuristiques. À l'heure où paraissent ses deux premiers numéros, la présentation générale de la revue sur OpenEdition Journals s'achève sur ces mots : « Nous publions des auteurs et acteurs prêts à objectiver, chroniquer et critiquer, au sens le plus riche du terme, l'évolution de leurs pratiques et de leur pensée. " Ce premier éditorial vise à expliciter succinctement ces intentions, l'historique du projet et les soutiens qui lui donnent les moyens de ses ambitions, tandis que léditorial suivant commentera le contenu de ces numéros.

Si une revue francophone d'humanités numériques voit le jour, après plusieurs années de genèse, c'est pour rendre disponibles et faire mieux connaître les travaux d'un champ de recherche aussi divers que dynamique. Publier en libre accès en est le moyen le plus efficace. C'est la contribution la plus élémentaire à la construction d'une science ouverte, dont l'éthique professionnelle et l'attention pratique aux cycles de données sont pour nous essentielles. Cependant, en donnant à lire les humanités numériques francophones, nous espérons aussi les " objectiver ", c'est-à-dire délimiter le champ par l'exemple. Nous souhaitons ainsi rendre perceptible la réalité des travaux, en mettant en va- 
leur des productions que la communauté des comités et des évaluateurs considère comme représentatives, utiles à partager à la fois pour illustrer et définir des méthodes transversales et pour donner un contenu à ce dont les modes et l'ubiquité des technologies numériques font parfois une boîte noire ou un signifiant flottant. "Chroniquer " les humanités numériques revient à les insérer dans une histoire collective, que ce soit par le réseau des noms, des dates et des lieux mentionnés ou bien par la rédaction d'articles délibérément consacrés aux trajectoires des acteurs et des actrices, à l'évolution des centres de recherche ou de formation, à des enquêtes fondées sur l'exploration des archives ou au recueil de la mémoire orale. "Critiquer ", enfin, c'est exercer la réflexivité qui fait tout l'intérêt des pratiques scientifiques et constitue un impératif épistémologique lorsque des sciences humaines et sociales désormais numérisées entendent cultiver des humanités numériques.

Ces orientations doivent s'intégrer aux fonctions habituelles d'une revue. Il s'agit naturellement de permettre l'archivage et la pérennité de ces échantillons d'une transformation en cours, de donner la possibilité d'une citation et l'assurance d'un référencement. Il s'agit aussi de légitimer les travaux des équipes, des chercheurs et chercheuses, ingénieures et ingénieurs dont les carrières portent ce renouvellement; de légitimer le champ des humanités numériques lui-même ; de confirmer la valeur $\mathrm{du}$ français comme vecteur de pensée et d'expression dans le domaine. Une revue peut et doit donner un espace à des controverses, avoir un rôle de recommandation et d'inspiration, travailler à la fédération et à l'animation d'une communauté.

De plus, ces fonctions recouvrent des enjeux à diverses échelles. Faire vivre le carrefour que sont les humanités numériques, c'est enrichir la pratique et la réflexion des participants, mais c'est également se donner les chances d'un développement plus intelligent au sein du champ universitaire et dans la société - ou plutôt dans les sociétés. En effet, le caractère francophone de la revue est une invitation à préciser et faire dialoguer les conditions singulières de cette évolution dans chaque pays ou territoire, non seulement en Europe et sur le continent nord-américain, mais aussi par exemple en Afrique.

\section{Une aventure collective}

Après un demi-siècle de relations moins intenses, plus sporadiques, entre l'informatique et les sciences humaines et sociales, le début du $\mathrm{XxI}^{\mathrm{e}}$ siècle a été marqué par une accélération et une institutionnalisation que le succès de l'étiquette "Digital Humanities " a matérialisées dans des pays anglophones - puis partout ailleurs, parfois avec des dénominations locales. La genèse de la revue est, de fait, profondément enracinée dans le développement européen et français des humanités numériques.

Un récit de fondation possible s'ouvrirait en 2010 à Paris, lors du premier THATCamp européen, sur une péniche, et durant la mise au point collaborative d'un Manifeste des Digital Humanities. L'événement et le texte étaient placés à la fois sous le signe d'une revendication concernant la politique des savoirs et leur réorganisation dans un monde devenu numérique et d'une joyeuse humeur anti-institutionnelle librement empruntée à la culture hacker. Un THATCamp, «The Humanities and Technology Camp », était en effet une rencontre auto-organisée, propice aux 
échanges informels entre disciplines et entre métiers, et il y en a eu plus de 300 dans le monde depuis 2008. Le projet de revue, esquissé notamment lors des THATCamp de Paris en 2012 et de Saint-Malo en 2013, discuté lors du THATCamp de Lyon en 2014, a accompagné la création de l'association francophone des humanités numériques, Humanistica, elle-même officiellement fondée en 2014 à Lausanne, en marge du colloque Digital Humanities annuel de l'Alliance of Digital Humanities Organizations (ADHO).

Il a fallu plusieurs années - dans des contextes locaux et nationaux où la place des humanités numériques s'inventait, au sein des séances de séminaire et des projets de recherche, des cours de master et des comités de sélection, des directions d'infrastructure et des agences de financement - pour que le comité de direction réuni en 2016 mette au point le modèle scientifique, les partenariats institutionnels et l'infrastructure humaine et technique de la revue. Le numéro francophone de la revue Digital Humanities Quarterly, publié en 2018 sous la direction de membres du comité de coordination d'Humanistica, s'est inséré dans ce calendrier comme une répétition générale, témoignant de la volonté d'ouverture des institutions internationales plus anciennes. Notre premier appel à contribution a été diffusé en 2017 et nous sommes très reconnaissants envers les auteurs et autrices qui nous ont fait confiance et ont suivi avec patience les étapes de cette aventure. L'éditorial du numéro 2 précise le fonctionnement prévu, au rythme de deux numéros par an.

La création de la revue est intimement liée à l'existence d'Humanistica, mais elle est également rendue possible par le soutien financier d'ADHO, organisation faîtière - pour employer l'expression suisse - dont l'association francophone est une constituent organisation. Nous devons beaucoup aussi à l'Institut des sciences humaines et sociales (INSHS) du Centre national de la recherche scientifique français (CNRS), qui nous soutient à travers un poste fondamental : le secrétariat d'édition, qui dépend en grande partie du travail d'une éditrice de la Maison des sciences de l'homme en Bretagne (MSHB). Un partenariat avec les Presses universitaires de Liège (PULg) nous a permis de réaliser le stylage des deux premiers numéros en vue de leur intégration à la plateforme d'OpenEdition.

Si les institutions donnent un cadre et une stabilité aux projets, les réseaux sont toujours aussi des réseaux humains. Nous avons déjà remercié les collègues qui nous ont confié des textes. Nous avions également annoncé aux évaluateurs et évaluatrices qu'une liste serait publiée sur le site de la revue pour leur témoigner notre gratitude et attester leur contribution. C'est chose faite. Nous perpétuerons cette saine habitude en mettant à jour cette page à chaque nouveau numéro. 


\section{Nommer un champ qui s'invente}

La revue porte le nom qui est actuellement le plus évident, car le plus courant, pour désigner les usages réflexifs des technologies numériques dans la recherche et l'enseignement en sciences humaines et sociales. Depuis le Manifeste des Digital Humanities de 2010, ou, peu avant et à l'initiative des mêmes personnes, l'ouverture à l'École des hautes études en sciences sociales d'un séminaire qui employait l'expression anglophone dans son intitulé, le syntagme " humanités numériques " s'est imposé en français.

Cet éditorial n'est pas le bon lieu pour nous interroger sur le sens de cette origine, l'histoire de ce calque, le décalage conceptuel qu'il peut impliquer, ni la question de savoir quelle serait la particularité d'humanités numériques faites et pensées en français. En revanche, nous voulons affirmer ici que nous assumons pleinement l'historicité du terme : dans des communautés en évolution, la revue suit l'usage, sans s'interdire de le critiquer, voire de l'orienter, parce que son principal souci est de rencontrer un public - vous ! - et de jouer son rôle de forum. Dans une époque où la pluralité épistémologique des SHS semble faire l'objet d'un consensus, et afin de nous intéresser au dialogue avec d'autres traditions scientifiques donnant une place plus centrale à la formalisation et aux méthodes quantitatives, il nous semble peu utile de chercher une définition stable et unique.

Ne faisons pas un fétiche d'une expression qui est davantage le nom d'un lieu qu'une description. Du reste, le contenu du mot « humanités » a varié historiquement, au gré des oppositions (étudier l'humain plutôt que le divin), des adjectifs accolés (selon que les humanités sont qualifiées de " classiques ", de " modernes " ou de " scientifiques ") et même des institutions (jusqu'à la reconnaissance officielle d'" humanités numériques " comme intitulé légitime de master). Le numérique, quant à lui, est une vaste gamme d'instruments et d'usages sociaux en interaction avec une informatique toujours plus diverse depuis qu'elle est devenue une force motrice de notre histoire. En réalité, depuis environ dix ans en français et quinze ans en anglais, parler d'humanités numériques, c'est désigner un mouvement de réflexion et de transformation des sciences humaines et sociales - les sciences humaines étant habituellement les moins directement et massivement poussées à intégrer en profondeur des méthodes informatiques ou à les inventer.

Le paradoxe de la définition se résout en marchant. L'oxymore de l'intitulé disparaît avec l'habitude, tout en nous réveillant périodiquement de notre sommeil dogmatique quant aux affordances de nos outils, à la pertinence de nos méthodes ou aux conditions pratiques des discours savants. À nos yeux, la multiplicité et l'ouverture du champ sont des avantages. Il y a des innovations spectaculaires et technologiques, d'autres plus discrètes et non moins réelles, orientées par exemple vers la négociation avec les connaissances accumulées dans un domaine ou sur un corpus. Toute conversation argumentée explicitant sa position dans le champ pourra convaincre de son intérêt nos évaluateurs - chacune et chacun d'entre vous, lecteurs, ayant potentiellement le statut d'auteur et d'évaluateur. 
Pour vous inviter à faire entendre vos idées et à faire connaître vos expériences, nous renouvellerons donc l'invitation que nous lancions aux participants d'un atelier préparatoire organisé aux prémices du projet : "Cette revue sera ce que nous en ferons, comme les humanités numériques. " Ce " nous » est inclusif. Cette revue est celle d'une communauté ouverte, dont l'avenir est encore à écrire.

Le comité de direction : Aurélien Berra, Emmanuel Château-Dutier, Sébastien Poublanc, Émilien Ruiz, Nicolas Thély (membres actuels), Emmanuelle Morlock (membre durant la préparation de ces numéros, jusqu'en octobre 2019)

\section{Droits d'auteur}

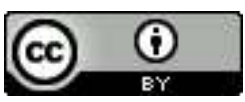

Les contenus de la revue Humanités numériques sont mis à disposition selon les termes de la Licence Creative Commons Attribution 4.0 International. 